\title{
Suppression of induction of experimental immune mediated blepharoconjunctivitis by tolerogenic conjugates of the antigen and monomethoxypolyethylene glycol
}

\author{
Atsuki Fukushima, Koji Nishino, Hironori Yoshida, Masaru Takata, Hisayuki Ueno
}

\begin{abstract}
Aim-Covalent conjugates consisting of diverse antigens coupled to optimal numbers of monomethoxypolyethylene glycol (mPEG) molecules have been shown to suppress antigen specific antibody formation. In this study, the possibility was examined that the same conjugates might prevent experimental immune mediated blepharoconjunctivitis (EC, formerly EAC) which had been shown to be caused by $\mathrm{CD}^{+} \mathrm{T}$ cells-that is, to cell mediated immunity.

Methods-6-8 week old male Lewis rats were used. The test groups of rats received two intravenous injections, each of $300 \mu \mathrm{g}$, of a conjugate of ovalbumin mPEG (OVA $(\mathrm{mPEG})_{11}$ ) in phosphate buffered saline (PBS), 14 and 28 days before the single immunisation with OVA in complete Freund's adjuvant. The rats were challenged 3 weeks later by eye drops containing OVA; 24 hours later they were sacrificed, and their eyes, blood, and lymph nodes were harvested for histological examination and determination of antiOVA antibody titres and levels of cellular immunity. Two control groups received PBS or OVA in PBS before immunisation. Furthermore, the possibility that OVA(mPEG) $)_{11}$ may have induced OVA specific suppressor cells was tested by establishing the effects of the co-transfer of splenocytes from OVA(mPEG) $)_{11}$ treated rats with OVA primed lymph node cells on the manifestations of EC.
\end{abstract}

Results-Either PBS or OVA pretreated rats, which had not received OVA(mPEG) $)_{11}$, developed high levels of antibodies and cell mediated immune responses to OVA, and application of eye drops led to blepharoconjunctivitis with massive cellular infiltration. In contrast, pretreatment with OVA(mPEG) $)_{11}$ prevented cellular infiltration into the lids and conjunctivas, as well as the formation of detectable humoral and cellular immunity against OVA. Co-transfer of splenocytes from OVA $(\mathrm{mPEG})_{11}$ treated rats with OVA primed lymph node cells suppressed the cellular infiltration on application of OVA on the conjunctiva.

Conclusions-These data indicate that intravenous injection of OVA(mPEG) conjugates suppressed both humoral and cellular immunity by the effects of antigen specific suppressor cells, thus leading to the inhibition of development of EC. (Br f Ophthalmol 1999;83:973-979)

Induction of antigen specific immunological tolerance is a most attractive strategy for treating the corresponding immunological diseases. Among experimental immunological therapeutic strategies, one may cite treatment with monoclonal anti-CD4 antibodies, ${ }^{1}$ antigen feeding, ${ }^{2}$ or injection of peptides representing the epitopes of the autoantigen in question. ${ }^{3}$ In addition to the encouraging results in animals, some of these procedures were demonstrated to be also effective in patients. ${ }^{45}$

Obviously antigen specific suppression of deleterious immune responses is to be preferred over the other procedures. In fact, suppression of primary immune responses has been achieved by different procedures - for example, by intravenous injection of (i) massive doses of aggregate free antigen, ${ }^{6}$ (ii) conjugates of antigen and autologous $\gamma$ globulins, ${ }^{7}$ (iii) recently by conjugates of the antigens in question and optimal numbers of molecules of polyethylene glycol (PEG). ${ }^{8}$

We established an disease model of blepharoconjunctivitis in Lewis rats utilising ovalbumin (OVA) as a model non-ocular antigen and termed experimental immune mediated blepharoconjunctivitis (EC, formerly EAC). ${ }^{9}$ This disease is transferable by in vitro stimulated lymph node cells from OVA primed rats $^{910}$ as well as OVA specific $\mathrm{CD}^{+}{ }^{+} \mathrm{T}$ cell line, ${ }^{11}$ whereas little, if any, IgE was detected in serum from antigen primed rats. In addition, a large amount of IFN- $\gamma$ was produced by lymph node cells and this cell line. ${ }^{11}$ These data indicated that EC is caused by Th1 type $\mathrm{CD} 4^{+} \mathrm{T}$ cells (and/or IgG antibodies), while IgE is not involved in the induction of EC.

Until recently, antigen monomethoxypolyethylene glycol (mPEG) conjugates have been used for specific suppression of antibody responses. However, in this study we demonstrated that antigen-(mPEG $)_{11}$ conjugates were also capable of suppressing cellular responses. Hence, having established that cellular immunity was dominantly involved in both induction and effector phases of EC in rats, ${ }^{9}{ }^{10}$ we investigated in the present study the effects of tolerogenic $\mathrm{mPEG}$ conjugates of the same antigenthat is, OVA, on the suppression of cell mediated blepharoconjunctivitis.
Accepted for publication 15 March 1999 


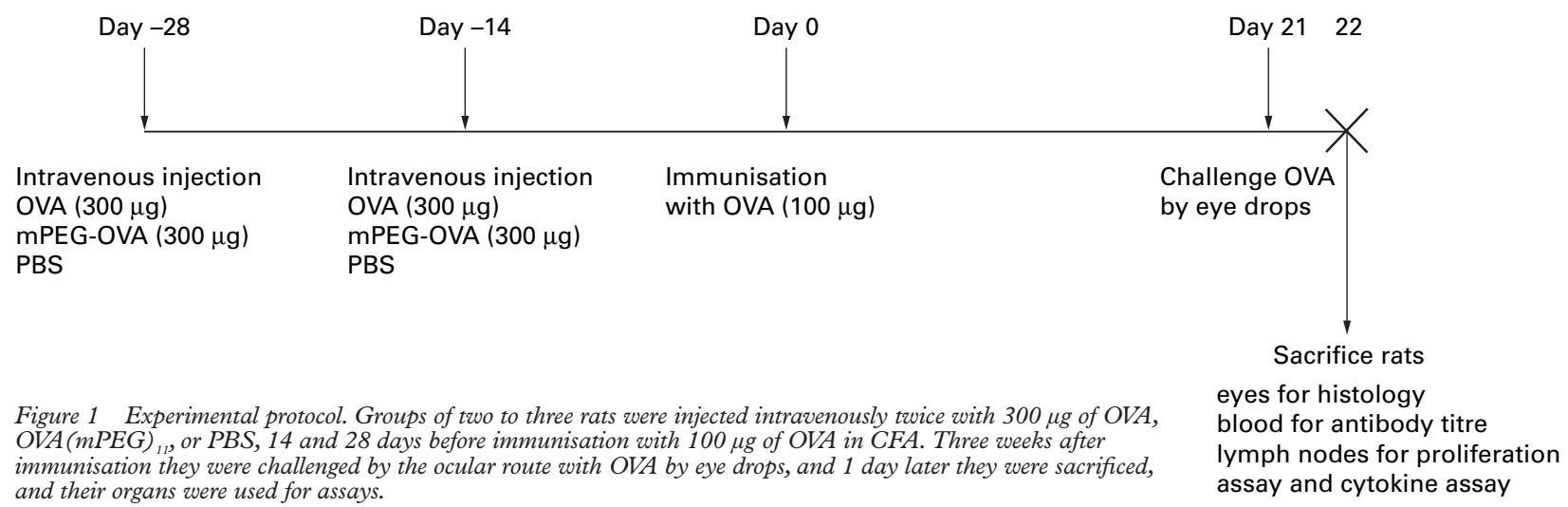

\section{Materials and methods}

RATS

Six to 8 week old male Lewis rats were purchased from Seac Yoshitomi, Fukuoka, Japan and were maintained in a pathogen-free animal facility at Kochi Medical School. All animal procedures conformed to institutional guidelines and to the ARVO resolution on use of animals in research.

PREPARATION OF THE TOLEROGEN BY COUPLING OVA TO MPEG

For the present study, mPEG (MW 5000) activated with cyanuric chloride and OVA (grade V) were purchased from Sigma Immunochemicals (St Louis, MO, USA) and converted to the corresponding conjugate $(\mathrm{OVA}(\mathrm{m}-$ PEG) ${ }_{11}$ ) using cyanuric chloride as the coupling agent, according to the procedure reported previously. ${ }^{12}$ There was no difference in endotoxin level between OVA and OVA(mPEG $)_{11}(2.3 \mathrm{ng} / \mathrm{ml})$.

SYNTHETIC OVA OVERLAPPING PEPTIDES Thirty eight overlapping 15-mer peptides, spanning the entire OVA sequence, were synthesised following the multipin method $^{13}$ based on the amino acid sequence of OVA (Peptide Institute, Inc, Osaka, Japan). Each peptide overlaps five amino acids.

\section{IMMUNISATION}

Each rat received in the left hind foot pad an injection of $100 \mu \mathrm{l}$ containing $100 \mu \mathrm{g}$ of OVA emulsified in complete Freund's adjuvant (CFA, Yatron, Tokyo, Japan). In some experiments, bovine serum albumin (BSA, Sigma Immunochemicals, St Louis, MO, USA) was used instead of OVA as both immunising and challenging antigen at the same dose and concentration to test whether the suppression is antigen specific or not.

EXPERIMENTAL PROTOCOL FOR TESTING THE IMMUNOSUPPRESSIVE CAPACITY OF OVA(MPEG) ${ }_{11}$ Pretreatment of rats with OVA $(m P E G)_{11}$ Figure 1 illustrates the experimental procedure used. Each rat received intravenously two injections of $500 \mu \mathrm{l}$, each containing $300 \mu \mathrm{g}$ of OVA(mPEG) $)_{11}, 28$ and 14 days before immunisation; each of two groups of control rats received $500 \mu \mathrm{l}$ of PBS or $300 \mu \mathrm{g}$ of immunising OVA in the same volume. Three weeks after immunisation, all rats were challenged with a total of $250 \mu \mathrm{g}$ of OVA by $50 \mu \mathrm{l}$ of eye drops, each drop consisting of a solution of $5 \mathrm{mg}$ of OVA per ml of PBS. Each group consisted of three rats in each experiment and each experiment was repeated four times.

Effects of splenocytes from OVA (mPEG) ${ }_{11}$ treated rats on the induction of $E C$ in rats receiving lymph node cells from third party immunised rats Cells from inguinal lymph nodes of OVA primed rats were harvested between 14 and 21 days after immunisation. These cells (100 $\times 10^{6}$ ) were cultured for 3 days in $25 \mathrm{~cm}^{2}$ flask (Nunc, Roskilde, Denmark) containing $10 \mathrm{ml}$ of RPMI1640 medium (Nikken, Osaka, Japan) supplemented with $2 \mathrm{mM}$ L glutamine, $5 \times 10^{-5}$ M 2-mercaptoethanol (2-ME), $100 \mathrm{U} / \mathrm{ml}$ penicillin, $100 \mu \mathrm{g} / \mathrm{ml}$ streptomycin, $50 \mu \mathrm{g} / \mathrm{ml}$ OVA, and $10 \%$ fetal calf serum (FCS, ICN Biomedical Japan Co, Tokyo, Japan). At the end of the 3 day period, the stimulated cells were harvested and washed three times. ${ }^{9}{ }^{10}$ The stimulated cells $\left(30 \times 10^{6}\right)$ were injected intravenously into syngeneic recipients either with $200 \times 10^{6}$ of splenocytes from naive rats, or from rats which had been treated with OVA(mPEG $)_{11}$. As described in the preceding paragraph, 4 days later the recipients' eyes were challenged by eye drops of the OVA solution, and 24 hours later their eyes were enucleated for histological examination.

\section{COUNT OF INFILTRATED CELLS IN THE}

CONJUNCTIVA

After clinical evaluation, the eyes including the lids were fixed in $10 \%$ buffered formalin and embedded in paraffin. Thick sections of $10 \mu \mathrm{m}$ were stained with haematoxylin and eosin, and the infiltrated cells were counted in the palpebral conjunctiva with an eye piece grid of $400 \times$ magnification. ${ }^{9}{ }^{10}$

ASSESSMENT OF CELLULAR RESPONSES

Cellular responses of immunised rats were measured by the lymphocyte proliferation assay. For each experiment, lymph nodes of each group of rats were combined. Lymphocyte proliferation assays were performed in quadruplicate utilising 96 flat bottom well plates. Lymph node cells $\left(3 \times 10^{5}\right.$ cells/well $)$ were cultured in a final volume of $0.2 \mathrm{ml} \mathrm{RPMI}$ medium supplemented with 5\% FCS. The cells were stimulated with (i) OVA or OVA(m- 

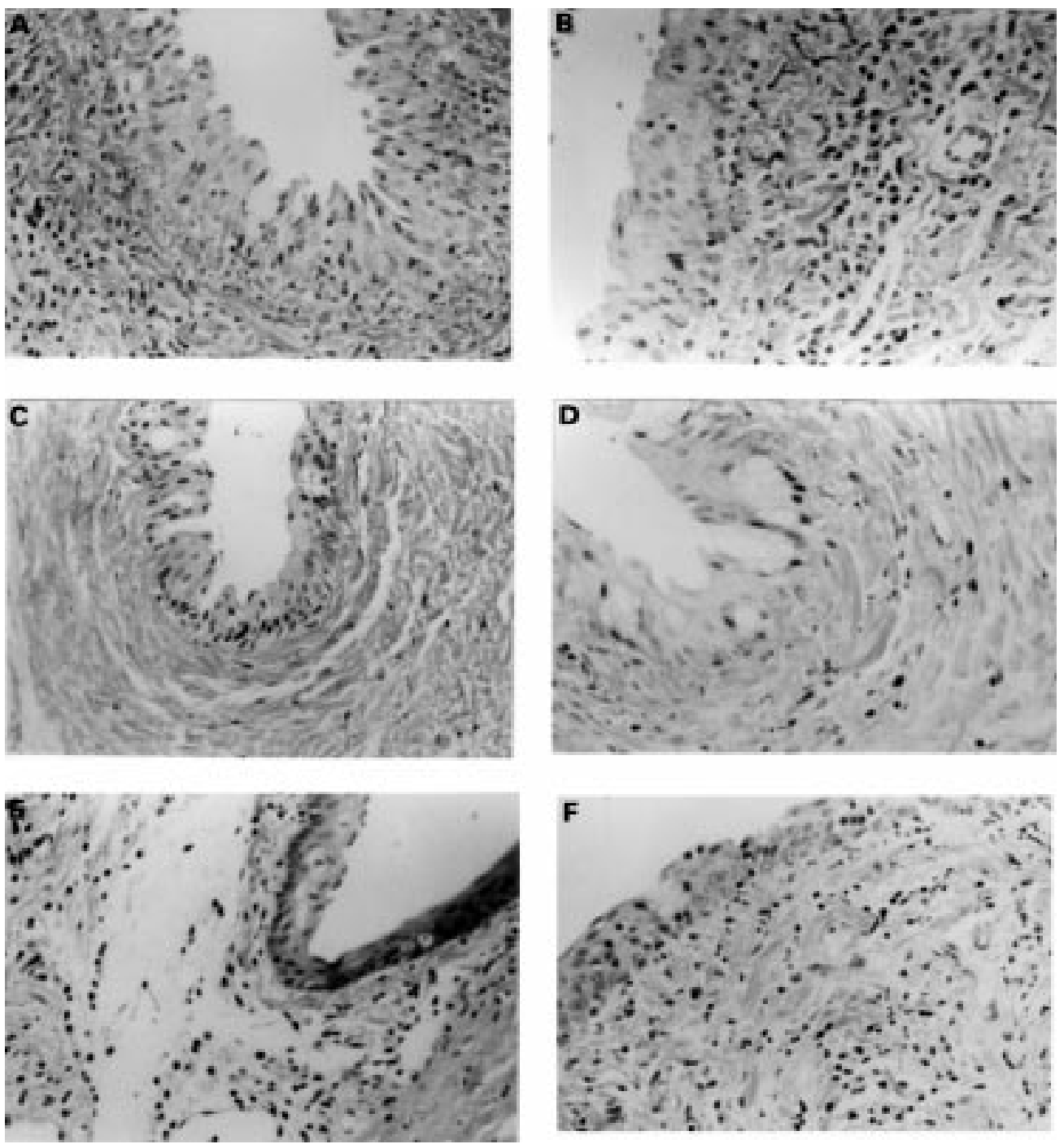

Figure 2 Histological sections of the conjunctiva from rats pretreated with $P B S(A, B), O V A(m P E G)_{11}(C, D)$, or OVA $(E, F)$. Sections were stained with haematoxylin and eosin. Magnifications were either $\times 160(A, C, E)$ or $\times 320(B, D, F)$.

PEG) $)_{11}$ at concentrations of $0.1,1,10,100$ $\mu \mathrm{g} / \mathrm{ml}$, (ii) synthetic OVA overlapping peptides at $20 \mu \mathrm{g} / \mathrm{ml}$, and (iii) purified protein derivatives (PPD, Japan BCG, Tokyo, Japan) at 5 $\mu \mathrm{g} / \mathrm{ml}$. After incubation of 72 hours at $37^{\circ} \mathrm{C}$ in a humidified atmosphere with $5 \%$ carbon dioxide, the cultures were pulsed for 16 hours with $0.5 \mu \mathrm{Ci} /$ well of ${ }^{3} \mathrm{H}$-thymidine (Japan Atomic Energy Research Institute, Tokai,

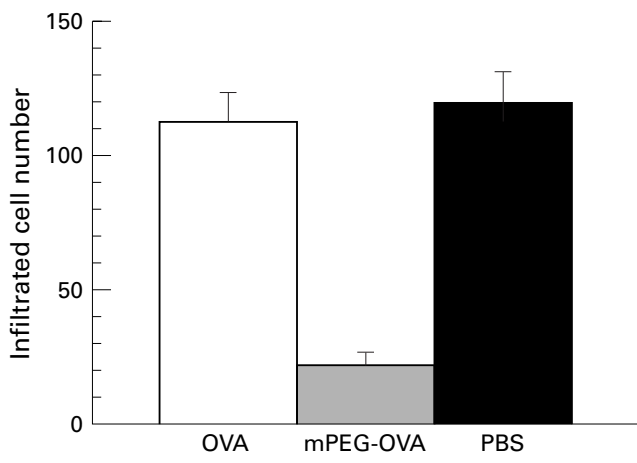

Figure 3 The infiltrated cell number refers to the average of infiltrated cells in the palpebral conjunctiva of rats treated with OVA, OVA (mPEG) , or PBS. The error bars indicate standard errors. Each group consisted of 12 rats.
Japan). The cultured cells were then harvested and their radioactivity was measured. The data are expressed as stimulation indices (SI = mean cpm in stimulated cultures/mean cpm in unstimulated control cultures) or delta cpm (mean cpm in stimulated cultures - mean cpm in unstimulated control cultures). In addition to cellular proliferative responses, production of IFN- $\gamma$ and IL- 4 by stimulation with OVA in the culture supernatant of lymph node cells from three different groups were tested using commercial cytokine ELISA (BioSource International, Camarillo, CA, USA) detailed in previous reports. ${ }^{1011}$

ASSESSMENT OF HUMORAL RESPONSES

Serum was collected from each rat via cardiac puncture at the time of sacrifice, and serum levels of anti-OVA antibodies were determined by direct ELISA. Briefly, OVA (500 ng/well) was adsorbed on to 96 well plates for 2 hours at $37^{\circ} \mathrm{C}$. The plates were washed with PBSTween and serially diluted serum samples were added to the wells. Thereafter, the plates coated with the antibody were treated with peroxidase conjugated anti-rat IgG and washed 


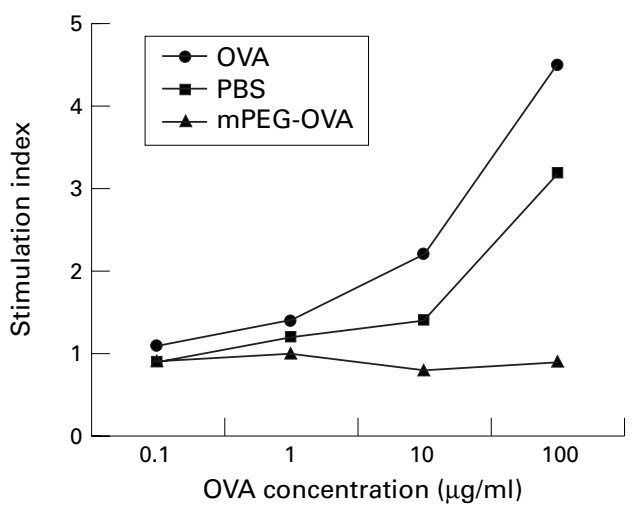

Figure 4 Proliferative response to OVA by lymph node cells of rats pretreated with OVA (mPEG) ${ }_{11}$, OVA, or PBS, and immunised with OVA. The draining lymph node cells were harvested and tested for their capacity to proliferate. The recorded data represent means of the values of stimulation indices (SI) of cells of three rats of each group. Similar results were obtained in three confirmatory experiments. The combined mean cpm values in the unstimulated control cultures of rats were between 465 and 503.

before addition of the substrate, 3,3',5,5'tetramethylbenzidine base (TMB-ELISA, Gibco BRL, Gaithersburg, MD, USA); finally the optical densities (ODs) of the contents of all wells were determined at $610 \mathrm{~nm}$. For detecting OVA specific IgE, the passive cutaneous anaphylaxis (PCA) method $^{14}$ was used.

\section{STATISTICAL ANALYSIS}

Statistical comparison in each group was performed by Student's $t$ test.

\section{Results}

INHIBITION OF DISEASE INDUCTION

As previously reported, ${ }^{9}$ the main feature of $\mathrm{EC}$ is mononuclear cellular infiltration in the conjunctiva. The severity of EC has been assessed by counting infiltrated cells in the palpebral conjunctiva, because severity of clinical grading was in parallel with the infiltrated cell number. ${ }^{9}$ As shown in Figure 2, massive cellular infiltration was demonstrated in PBS and OVA treated rats, while minimal inflammatory cells were detected in OVA(mPEG) $)_{11}$ treated rats. The number of cells infiltrated on challenge with OVA into the palpebral conjunctivae of rats, which had been pretreated with PBS, OVA, or OVA(mPEG) $)_{11}$, are shown in Figure 3. The infiltrated cell number of the group with OVA (average infiltrated cell number $(n)=113$ ) was almost identical to that of the control group $(n=120)$. By contrast, however, $\mathrm{n}$ for the group treated with OVA(mPEG) $)_{11}$ was only 22 -that is, it was significantly lower than that for the two other groups $(p<0.01)$. No differences in inflammatory cell numbers in the conjunctiva among three groups of different treatment were detected in cases where BSA was used as an immunising and challenging antigen (data not shown).

INHIBITION OF CELLULAR AND HUMORAL RESPONSES IN RATS PRETREATED WITH OVA(MPEG) $)_{11}$

To investigate the effects of treatment of OVA(mPEG $)_{11}$ on the immune responses, we tested the levels of (i) cellular immunity by the proliferation assay or cytokine production (IFN- $\gamma$ and IL-4) of lymph node cells, and (ii) antibody responses by ELISA or PCA using the sera. Representative data of three rats per group were presented in Figures 4-6. Both cellular proliferation (Figs 4 and 5) and IgG production (Fig 6A) in response to OVA were depressed in rats pretreated with $\mathrm{OVA}(\mathrm{m}-$ PEG) $)_{11}$, in relation to the levels of rats treated with OVA or PBS. Inhibition of proliferative responses was remarkable in immunodomi-

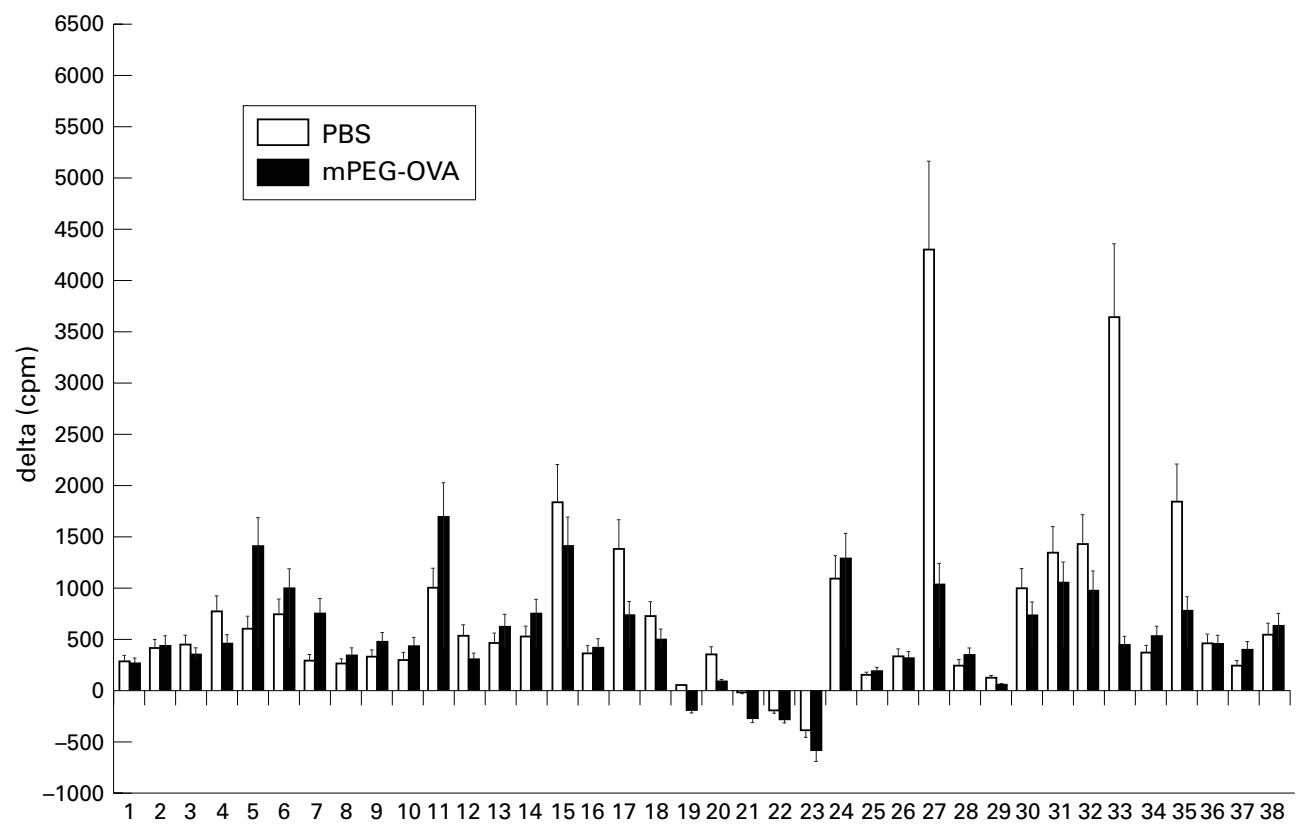

Figure 5 Proliferative response to OVA peptides by lymph node cells of rats pretreated with OVA(mPEG) ${ }_{11}$ or PBS, and immunised with OVA. The recorded data represent means of the values of stimulation indices (SI) of cells of two rats of each group. Similar results were obtained in one more experiment. The combined mean cpm values in the unstimulated control cultures of rats were $950(P B S)$ and $1130\left(O V A(m P E G)_{11}\right)$. 

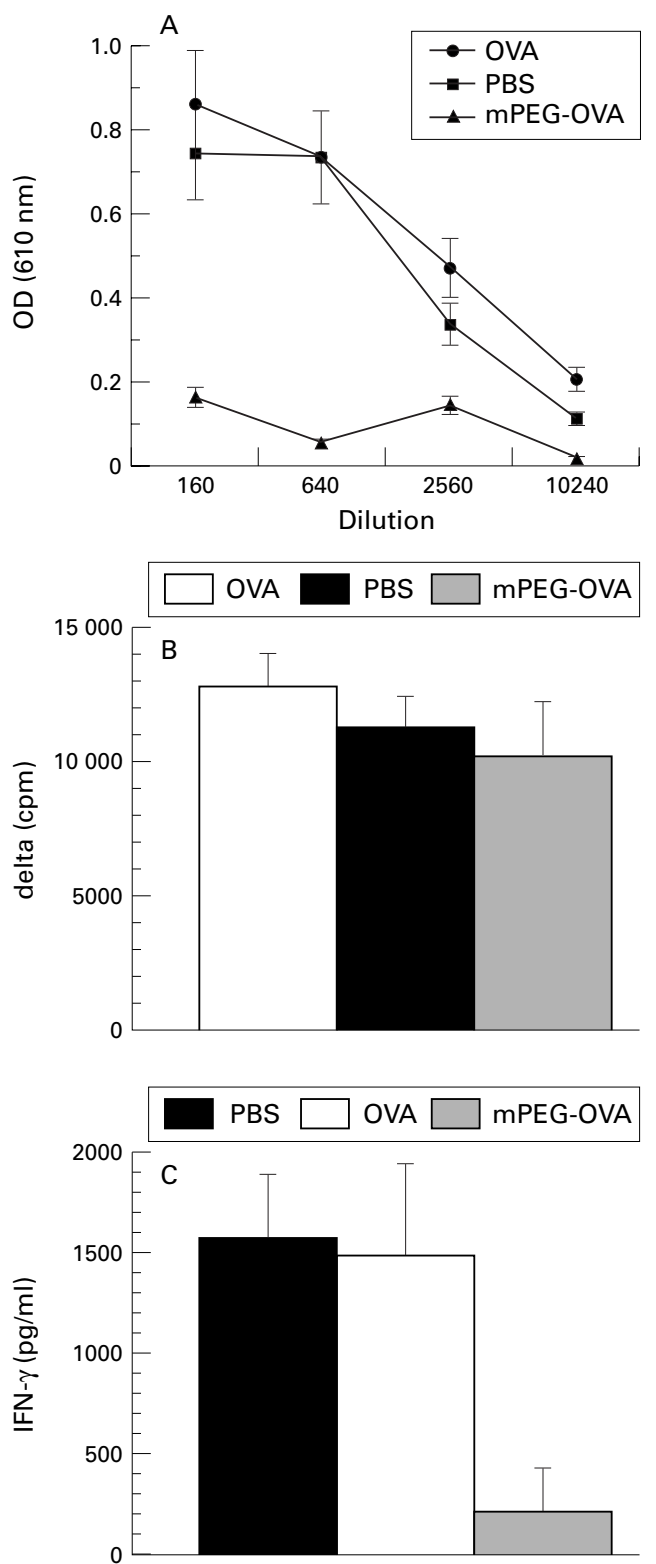

Figure 6 (A) Measurement of OVA specific IgG by ELISA in serum of rats pretreated with OVA (mPEG) ${ }_{11}$, $O V A$, or PBS, and immunised with OVA. The sera were collected at the time of sacrifice and assayed by ELISA. The abscissa indicates the degree of dilution of the serum; the ordinate represents absorbance at $610 \mathrm{~nm}$. Similar results were obtained in three other separate experiments. (B) Proliferative responses against PPD by lymph node cells of rats pretreated with OVA (mPEG), OVA, or PBS, and subsequently immunised with OVA. Except for using PPD instead of OVA, the procedure used was the same as employed for the experiment illustrated in Figure 4; in addition, the concentration of PPD was $5 \mu \mathrm{g} / \mathrm{ml}$. (C) Production of IFN- $\gamma$ in the culture supernatant of OVA primed lymph node cells from rats pretreated with OVA (mPEG) ${ }_{11}$, OVA, or PBS by in vitro stimulation with OVA. $Y$ axis indicates the produced amount of IFN- $\gamma$ $(\mathrm{pg} / \mathrm{ml})$.

nant epitopes (Fig 5). There were a few epitopes which exhibited higher stimulatory effects in response to OVA(mPEG) $)_{11}$ treated cells than PBS treated cells (Fig 5). By contrast, no significant differences in proliferative responses to PPD were noted among three groups $(0.248<\mathrm{p}<0.480)$ (Fig 6B). Lymph node cells from all three groups did not exhibit any apparent proliferative responses against

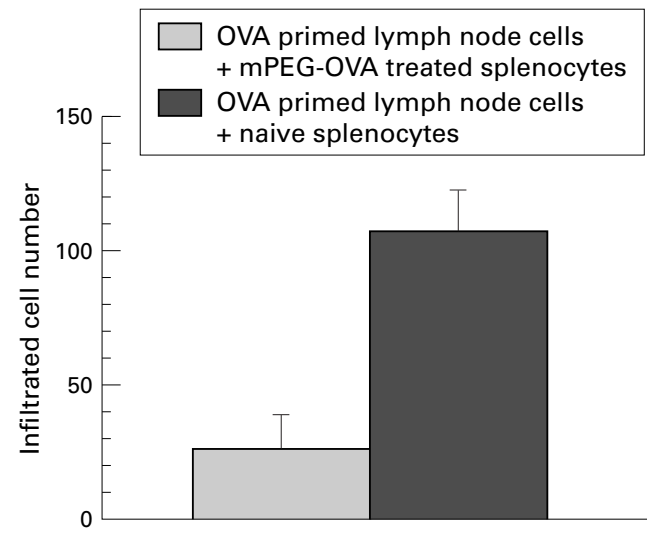

Figure 7 Infiltrated cell number in the palpebral conjunctivas of rats which received stimulated OVA primed cells, and either naive splenocytes or splenocytes from rats pretreated with OVA (mPEG) and subsequently treated with OVA in CFA (Fig 1). Stimulated OVA primed cells were transferred into recipients with splenocytes from either naive rats or rats treated with OVA (mPEG) ${ }_{11}$. Four days after cell transfer, the rats were challenged and 24 hours later they were sacrificed for counting infiltrated cells in the conjunctiva.

OVA(mPEG) $)_{11}$ (data not shown). In addition to cellular proliferative responses, production of IFN- $\gamma$ in the culture supernatant was suppressed in OVA(mPEG) $)_{11}$ treated groups compared with the other two groups $(\mathrm{p}<0.01)$ (Fig 6C). It is of note that IL-4 and IgE were below detectable levels by the assays used here in all three groups (data not shown).

CO-TRANSFER OF SPLENOCYTES OF OVA(MPEG $)_{11}$ TREATED RATS SUPPRESSED THE INDUCTION OF EC BY TRANSFER OF OVA PRIMED LYMPH NODE CELLS

To analyse the mechanism of tolerance induced by treatment with OVA(mPEG) ${ }_{11}$, splenocytes from OVA(mPEG) ${ }_{11}$ treated rats were passively transferred together with OVA primed lymph node cells, as outlined in the Materials and methods section. The data clearly show that the co-transfer of OVA(mPEG) $)_{11}$ treated splenocytes suppressed the influx of inflammatory cells into the conjunctivas compared with the co-transfer of splenocytes from naive rats $(\mathrm{p}=0.040)$ (Fig 7).

\section{Discussion}

The published studies to date support the conclusion that administration of a conjugate of a given antigen and an optimal number of mPEG molecules induces suppression of antibodies specific to the antigen in question. As reported previously, ${ }^{9}$ in our experimental model of blepharoconjunctivitis, cellular immunity, especially Th1 type cell mediated immunity, appeared to be essential both in the induction and effector phases of this disease. Hence, the aim of this study was to investigate the possibility that administration of $\mathrm{mPEG}$ conjugates of the corresponding antigen would inhibit both the induction of antigen specific cellular immunity and the consequent development of the disease.

Indeed, the results of this study supported the conclusion that pretreatment of rats with OVA(mPEG) $)_{11}$ downregulated the induction of 
both cellular and humoral immunity to OVA (Figs 4-6), and consequently inhibited development of OVA specific EC (Figs 2 and 3). To test the possibility that the tolerance demonstrated here by OVA(mPEG) $)_{11}$ treatment might be due to a change in the presentation of peptides, proliferative responses against OVA fragments were performed to compare with those treated with PBS. No fragments were selected to be stimulatory for lymph node cells from OVA(mPEG) $)_{11}$ treated rats (Fig 5), indicating that this suppression may not be epitope specific but rather protein specific. Interestingly, OVA(mPEG) $)_{11}$ did not have stimulatory effects to lymphocytes sensitised with OVA from rats pretreated with OVA(mPEG) $)_{11}$ intravenously. It is important to point out that whereas in vitro proliferative responses to OVA of lymph node cells from OVA(mPEG) ${ }_{11}$ treated rats were inhibited, the reactivity of these cells to PPD was the same as that of lymph node cells from OVA treated rats or from the control group (Fig 6A). In addition, the results that pretreatment with $\operatorname{OVA}(\mathrm{m}$ $P E G)_{11}$ did not suppress inflammatory cellular infiltration in the conjunctiva when a third party antigen BSA was used as an immunising and challenging antigen, further support the conclusion that antigen-mPEG conjugates suppress the immune response in an antigen specific manner.

Although some reports claim that intravenous injection of native antigen could induce tolerance, ${ }^{15} 16$ this statement is not supported by our attempt to induce tolerance by injection of the native antigen, OVA (Figs 4-6). Since this effect may be dependent on the dose and/or the physical state of antigens injected, we investigated the effect of different doses by intravenous injection of OVA, $2 \mu \mathrm{g}, 200 \mu \mathrm{g}$, and $20 \mathrm{mg}$, in three groups of rats, following the protocol shown in Figure 1. No differences in either proliferative responses or disease gradings (data not shown) were observed among these three groups in relation to the control group treated with PBS. Therefore, it is clear that it is difficult, if at all possible, to induce tolerance by pretreatment with OVA, compared with the successful suppression of the antigen specific immune response by $\mathrm{mPEG}$ antigen conjugates.

Recently, it was demonstrated that the development of diseases induced by Th1 type immune responses such as experimental autoimmune uveoretinitis (EAU) could be inhibited by augmentation of antigen specific Th2 type immune responses. ${ }^{17}{ }^{18}$ To investigate the possible involvement of augmentation of OVA specific Th2 type immunity by OVA(mPEG) treatment, IL-4 production by lymph node cells and IgE in the serum were tested. Neither IL-4 or IgE was detected in OVA(mPEG) treated rats by the assays used here suggesting that a systemic shift from Th1 type to Th2 type was not likely to be involved in the tolerance induced by treatment with $\operatorname{OVA}(\mathrm{mPEG})_{11}$. Although production of IL-4 was not tested in splenocytes from OVA(mPEG) $)_{11}$ treated rats because these transferred cells were injected without culture in vitro, it would be important and interesting to investigate whether these splenocytes are able to produce immunoregulatory cytokines such as IL-4 or TGF- $\beta$.

Using the experimental protocol shown in Figure 1, it is not possible to establish unequivocally if cellular immunity is involved in the development of the disease, and if the inhibitory effects of treatment with OVA(mPEG) $)_{11}$ affect mainly the cellular arm of the immune response. To shed some light on this question, we used a cell transfer system for determination of the involvement of cellular immunity. The development of disease, which was induced by transfer of OVA primed lymph node cells, was suppressed by co-transfer of splenocytes from OVA(mPEG) $)_{11}$ treated rats (Fig 7). This evidence may be interpreted as indicating that splenocytes from rats, which had been treated with OVA(mPEG) $)_{11}$, inhibited the manifestation of the disease which would have been induced by the exclusive transfer of OVA primed lymph node cells. Therefore, the proposed mechanism for induction of tolerance by pre-administration of antigen-mPEG conjugates postulates that antigen specific suppressor cells and/or their suppressive factors inactivate the corresponding antigen specific $\mathrm{T}$ helper cells. ${ }^{19}$ The data about suppression in the effector phase (Fig 7) might be interpreted as being that this suppression was effective at the challenge site, while the data during the induction phase (Figs 2 and 3) could be interpreted as being that this suppression was induced in the draining lymph nodes.

In conclusion, this study (i) confirms that successful induction of antigen specific tolerance may be achieved by antigen-mPEG conjugates, and (ii) extends this capacity of mPEG conjugates to suppression of antigen specific $\mathrm{T}$ cells as demonstrated by inhibition of the development of EC by these cells.

A Fukushima and $\mathrm{K}$ Nishino contributed equally to the study. We should like to thank Professor Alec H Sehon for his inspiration, helpful discussions, and critical editorial suggestions during the preparation of this manuscript; Professor Shigeyoshi Fujimoto and Dr Glen Lang for valuable suggestions; and Ms . logical slides.

1 Cobbold SP, Qin S, Leong LYW, et al. Reprogramming the immune system for peripheral tolerance with $\mathrm{CD} 4+$ and CD8+ monoclonal antibodies. Immunol Rev 1992;129:165-201.

2 Nussenblatt RB, Caspi RR, Mahdi R, et al. Inhibition of $\mathrm{S}$-antigen induced experimental autoimmune uveoretinitis
by oral induction of tolerance with S-antigen. F Immunol 1990;144:1689-95.

3 Kuchroo VK, Greer JM, Kaul D, et al. A single TCR antagonist peptide inhibits experimental allergic encephaantagonist peptide inhibits experimental allergic encephanol 1994;153:3326-36.

4 Nussenblatt RB, Gery I, Weiner HL, et al. Treatment of uveitis by oral administration of retinal antigens: results of a phase I/II randomized masked trial. Am $\mathcal{f}$ Ophthalmol 1997; 123:583-92.

5 Weiner HL, Mackin GA, Matsui M, et al. Double-blind pilot trial of oral tolerization with myelin antigens in multiple sclerosis. Science 1993;259:1321-4.

6 Chiller JM, Romball CG, Weigle WO. Induction of immunological tolerance in neonatal and adult rabbits. Differences in the cellular events. Cell Immunol 1973;8:2839.

7 Walters CS, Moorhead JW, Claman HN. Immunity and tolerance to hapten (NIP) coupled to an isologous carrier (mouse gamma globulin). F Exp Med 1972;136:546-55.

8 Lee WY, Sehon AH. Abrogation of reaginic antibodies with modified allergens. Nature 1977;267:618-9.

9 Fukushima A, Yoshida H, Iwamoto $\mathrm{H}$, et al. The role of cellular immunity both in the induction and effector phases of 
experimental allergic blepharo-conjunctivitis (EAC) in

rats. Exp Eye Res 1997;65:631-7.
10 Yoshida H, Yoshida O, Iwamoto H, et al. Analysis of effects of stimulation in vitro of ovalbumin (OVA) primed lymph node cells on adoptive transfer of experimental immunemediated blepharoconjunctivitis (EC) in Lewis rats. $\mathrm{Br} \mathcal{F}$ Ophthalmol 1998;82:1189-94.

11 Fukushima A, Nishino K, Yoshida O, Ueno H. Characterization of the immunopathogenic responses to ovalbumin (OVA) peptide 323-339 in experimental immunemediated blepharoconjunctivitis (EC) in Lewis rats. Curr Eye Res 1998;17:763-9.

12 Jackson CC, Charlton JL, Kuzminski K, et al. Synthesis, isolation and characterization of conjugates of ovalbumin with monomethoxypolyethylene glycol using cyanuric chloride as the coupling agent. Anal Biochem 1987;165:114-27.

13 Roman E, Harris DP, Jurcevic S, et al. H-2-associated effects of flanking residues on the recognition of a permissive mycobacterial T-cell epitope. Immunology 1995;86:183-9.

14 Mota I. On the site of rat reagin formation. Immunology 1966;11:137-40.
15 Gery I, Waksman BH. Role of the thymus in tolerance: V. Suppressive effect of treatment with nonaggregated and aggregated bovine gamma-globulin on specific immune responses in normal adult rats. F Immunol 1967;98:446-60. 6 Rauch HC. Modulation of adoptive cell transfers in experimental allergic encephalomyelitis by antigen treatment of donors. Cell Immunol 1981;60:220-7.

7 Saoudi A, Kuhn J, Huygen K, et al. TH2 activated cells prevent experimental autoimmune uveoretinitis, a TH1dependent autoimmune disease. Eur f Immunol 1993;23: 3096-103.

18 Kezuka T, Sakai J, Yokoi H, et al. Peptide-mediated suppression of experimental autoimmune uveoretinitis in mice: development of a peptide vaccine. Int Immunol 1996; 8:1229-35.

19 Bitoh S, Takata M, Maiti PK, et al. Antigen-specific suppressor factors of noncytotoxic CD8+ suppressor $\mathrm{T}$ cells downregulate antibody responses also to unrelated antigens when the latter are presented as covalently linked adducts with the specific antigen. Cell Immunol 1993;150: adducts 